\section{EVALUATION OF RADIOGRAPHIC RESPONSE IN THE INTACT RENAL MASS (INTACT-RMASS) TO IMMUNE CHECKPOINT INHIBITOR (ICI) COMBINATION REGIMENS IN PATIENTS WITH METASTATIC RENAL CELL CARCINOMA (MRCC)}

${ }^{1}$ Hamid Emamekhoo*, 'Danubia Hester, ${ }^{2}$ Saqib Abbasi, 'Jens Eickhoff, ${ }^{3}$ Tristan Bice, ${ }^{4}$ Luna Archaya, ${ }^{5}$ Ellen Jeager, ${ }^{6}$ Moshe Ornstein, ${ }^{1}$ Ali Pirasteh, ${ }^{5}$ Pedro Barata, ${ }^{4}$ Yousef Zakharia, ${ }^{3}$ Deepak Kilari, ${ }^{7}$ Elizabeth Wulff-Burchfield, ${ }^{1}$ Christos Kyriakopoulos. ${ }^{1}$ University of Wisconsin - Madison, Madison, WI, USA; ${ }^{2}$ University of Kansas Medical Center, Kansas City, KS, USA; ${ }^{3}$ Medical College of Wisconsin, Milwaukee, WI, USA; ${ }^{4}$ University of lowa, lowa City, IA, USA; ${ }^{5}$ Tulane Cancer Center, New Orleans, LA, USA; ${ }^{6}$ Cleveland Clinic, Clevelenad, OH, USA; ${ }^{7}$ Kansas University Medical Center, Kansas City, KS, USA

Background As most of the patients previously enrolled in trials had nephrectomy before starting systemic treatment (syst$\mathrm{Rx}$ ), the response of the intact-Rmass to novel ICI and tyrosine kinase inhibitor (TKI) combination regimens is not well described.

Methods A retrospective review of 227 patients with mRCC who were treated with ICI (single agent or combinations) in the 1st- or 2 nd-line was conducted. Following the appropriate regulatory process, collaborators from 6 US sites collected clinical, pathological, and outcome data via chart review. Overall response was investigator-assessed for all patients with at least one post-treatment scan or evidence of clinical progression after treatment initiation. Overall radiographic response (ORR) represents any radiographic response in the metastatic disease per investigator's assessment. To accurately assess response in intact-Rmass, 3-dimensional measurement of the intact-Rmass was performed and Rmass volume was calculated at baseline and at the time of best overall response for 1st- and 2nd-line therapy. Radiographic response in intactRmass is defined as $>30 \%$ decrease in the Rmass volume.

Results Median age at diagnosis was 62 years, $69 \%$ were male, $82 \%$ had clear cell histology. $15 \%$ and $12 \%$ had sarcomatoid and rhabdoid features, respectively. Overall, 82 patients (36\%) had a measurable intact-Rmass while receiving syst-Rx. 63 (28\%) patients never had a nephrectomy, and 10 (4\%) patients had delayed nephrectomy after a good overall response to syst-Rx. 108 (48\%) received ICI in 1st-line (88/ 108 received ipilimumab/nivolumab combination). 91 (40\%), and $18(8 \%)$ patients received TKI, or ICI+TKI in 1st-line. $161(71 \%)$ and $86(38 \%)$ of the patients received 2nd-line and 3rd-line therapy, respectively. $104(46 \%)$ received ICI in 2nd-line (75/104 treated with single-agent ICI). 48 (21\%), and $4(2 \%)$ patients received TKI, or ICI+TKI in 2 nd-line. Radiographic response in intact-Rmass for evaluable patients is summarized in table 1 . The highest response rates in intact-Rmass were seen with ICI+TKI combinations. Higher rates of radiographic response in intact-Rmass were seen in patients treated with ICI in 1st-line compared to 2nd-line, possibly related to higher usage of ICI combinations (ipilimumab/nivolumab) in 1st-line. Overall metastatic disease response to different regimens in the 1st-line or 2nd-line was not different based on the history of nephrectomy prior to syst-Rx (table 2).

Abstract 294 Table 1 Radiographic response ( $\geq 30 \%$ decrease in volume) in the intact renal mass

\begin{tabular}{|l|l|l|l|}
\hline & ICI & TKI & ICI+TKI \\
\hline $1^{\text {st }}$ Line systemic treatment & $32 / 108(30 \%)$ & $23 / 91(25 \%)$ & $10 / 18(56 \%)$ \\
\hline $2^{\text {nd }}$ Line systemic treatment & $10 / 104(10 \%)$ & $18 / 48(48 \%)$ & $2 / 4(50 \%)$ \\
\hline
\end{tabular}

Abstract 294 Table 2 Overall radiographic response (ORR) per investigator assessment

\begin{tabular}{|l|l|l|l|}
\hline & $\begin{array}{l}\text { No previous } \\
\text { nephrectomy }\end{array}$ & $\begin{array}{l}\text { Had previous } \\
\text { nephrectomy }\end{array}$ & p-value \\
\hline $\mathrm{ICl}-1^{\text {st line }}$ & $10 / 37(27 \%)$ & $25 / 71(35 \%)$ & 0.39 \\
\hline $\mathrm{ICl}-2^{\text {nd }}$ line & $6 / 20(30 \%)$ & $11 / 84(13 \%)$ & 0.09 \\
\hline $\mathrm{TKI}-1^{\mathrm{st}}$ line & $6 / 15(40 \%)$ & $23 / 76(30 \%)$ & 0.55 \\
\hline $\mathrm{TKI}-2^{\text {nd }}$ line & $3 / 19(16 \%)$ & $10 / 29(34 \%)$ & 0.15 \\
\hline $\mathrm{ICl}+\mathrm{TKI}-1^{\text {st }}$ line & $6 / 10(60 \%)$ & $3 / 8(38 \%)$ & 0.64 \\
\hline $\mathrm{ICl}+\mathrm{TKI}-2^{\text {nd }}$ line & $1 / 3(33 \%)$ & $0 / 1(0 \%)$ & 0.99 \\
\hline
\end{tabular}

Conclusions Higher radiographic response rates in the intactRmass were seen in patients treated with ICI+TKI and ICI in the 1st-line. There was no significant difference in overall metastatic disease response to 1st- or 2nd-line treatment based on the history of nephrectomy prior to syst-Rx.

Ethics Approval Each of the 6 participating centers had their IRB approved protocol for retrospective study and data collection. Data Use Agreements were obtained for each center to share limited data set data with University of Wisconsin Madison (IRB protocol UW17148 \# 2018-0213). Final analysis was performed at University of Wisconsin.

Consent not applicable to retrospective studies.

http://dx.doi.org/10.1136/jitc-2021-SITC2021.294 